\title{
How sugars are sabotaging our health
}

\section{Alexander Frame}

\section{University of British Columbia}

In Canada, obesity has nearly doubled from 1978 to 2008. In 2008, based on Body Mass Index, the proportion of obese and overweight individuals over the age of 18 was $25.3 \%$ and $36.8 \%$, respectively (Table1). ${ }^{1}$ Thus, an alarming $62.1 \%$ of the adult population was at an unhealthy weight. The increased availability of processed foods and beverages shows a close temporal relationship with the epidemics of obesity and type 2 diabetes.

Obesity is significantly associated with higher risk for several medical conditions: type 2 diabetes, cancer, hypertension, heart failure and stroke (Table 1). ${ }^{1}$ The estimated annual Canadian healthcare costs associated with obesity vary between $\$ 4.6$ and $\$ 7.1$ billion. ${ }^{1}$ Given the escalating morbidity, mortality and health expenditures of obesity linked diseases, preventative measures have never been more vital. A survey of dietary trends and how carbohydrates, and specifically sugars are metabolized, should focus a response to this serious epidemic.

\section{Dietary Trends}

The consumption of processed foods and beverages has climbed steadily over decades. Mozaffarian (2011) made note of US dietary trends between 1965 and 2002, positively associating the consumption of refined sugars and starches and sugar-sweetened beverages with weight gain. ${ }^{2}$ During this period the proportion of calories from beverages increased from $11.8 \%$ to $21.0 \%$ of total calories. ${ }^{2}$ Conversely, the consumption of dairy products and unprocessed foods such as nuts, whole grains and vegetables were negatively associated with weight gain. ${ }^{2}$ Given the rise in refined sugar and processed food intake, an examination of metabolism could guide necessary action.

\section{Carbohydrate Metabolism}

Most carbohydrates, with the exception of fiber, are

\begin{tabular}{|lll|}
\hline \multicolumn{3}{l}{ Table 1: Canadian population divided according to Body Mass Index } \\
\hline Classification & BMI $\left(\mathrm{kg} / \mathrm{m}^{2}\right)$ & Risk of Other Conditions \\
\hline Underweight & $<18.5$ & Mildly increased \\
Normal & $18.5-24.9$ & Average \\
Overweight & $25.0-29.9$ & Mildly increased \\
Obese Class 1 & $30-34.9$ & Moderate \\
Class 2 & $35-39.9$ & Severe \\
Class 3 & $\geq 40.0$ & Very severe \\
\hline
\end{tabular}

metabolized as fructose and/or glucose, the main sugar building blocks. When physiological doses of carbohydrates are consumed, homeostasis is maintained with resulting normal blood sugars, blood pressure and lipid profiles. However, with the advent of processed foods high in starch and beverages high in sugar the consumption of carbohydrates has reached pharmacological doses. It is difficult to quantify physiologic and pharmacologic doses given individual variability in insulin resistance and carbohydrate metabolism. However, the Institute of Medicine noted the range of median carbohydrate intakes of 220-330 g/day dramatically exceed minimum recommendations of $130 \mathrm{~g} /$ day. ${ }^{3}$ Increasing evidence shows carbohydrate metabolism is altered in obesity leading to a cascade of maladaptive lipid metabolism.

With the over-consumption of refined sugars, starches, and refined grains the body diverts excess calories towards triglyceride production via liver lipogenesis. Fructose, under physiologic conditions, is metabolized into glycogen and some is broken down for energy. Any remaining fructose has been shown to increase production of proatherogenic lipoproteins - a result of excess triglyceride production. ${ }^{4}$ Glucose is utilized at approximately 50 calories per hour at rest; glucose not utilized must be stored as glycogen or converted into triglycerides. The consumption of a low-fat calorie balanced diet with a high glucose and/ or starch content such as potatoes or pasta stimulates liver lipogenesis, increasing serum triglycerides even in normal weight individuals. ${ }^{5}$ Additional evidence supports 
C the association of high fructose consumption with hypertension, dyslipidemia, visceral adiposity, increased insulin resistance, and increased inflammation. ${ }^{4}$ Thus, excess fructose and glucose have similar health impacts dyslipidemia and obesity.

The connection between dyslipidemia and obesity is insulin resistance and ensuing hyperinsulinemia. Adipose tissue in obesity contain high amounts of inflammatory cells and releases elevated amounts of inflammatory mediators precipitating insulin resistance. ${ }^{6}$ With insulin resistance being proportional to the amount of central adipose tissue, the body compensates with relative elevations in insulin. ${ }^{6}$ Both promote liver lipogenesis, by diverting consumed sugars into triglyceride production and elevating apolipoproteins, transport proteins essential for triglyceride transport within the blood. With a high carbohydrate diet there is predominance for proatherogenic lipoproteins. ${ }^{7}$ The resulting dyslipidemia with inflammation is one manifestation of a pharmacological dose.

\section{Intervention}

Fortunately, the abnormalities associated with obesity and excess sugar consumption can be controlled and reversed. Although voluntary calorie restricted diets can be effective, they first require some degree of weight loss to reduce insulin resistance and improve lipid profiles. ${ }^{8}$ Moreover, they are difficult to maintain, with rebound being common. However, a low carbohydrate diet is not calorie restricted, does not require weight loss to show near immediate reductions in liver lipogenesis followed by improved lipid profiles, and re-establishes normal carbohydrate homeostasis. ${ }^{8}$ Research has shown that significant satiety and weight loss are associated with the consumption of dairy, nuts, protein and foods high in fiber, and that weight gain results from the consumption of refined sugars and starches and sugar-sweetened beverages. ${ }^{2,4,9}$ Lowering the intake of simple sugars and carbohydrates broken down into simple sugars - pasta, bread, potatoes and white rice - increasing dairy, nuts, protein and fiber rich carbohydrates and allowing for moderate increases in fat would reduce liver lipogenesis and re-establish normal homeostasis including control of caloric intake. Although this necessitates exchanging some carbohydrates for fats, their addition has been shown not to adversely affect lipid profiles or increase risks for chronic disease. ${ }^{10}$

\section{Acknowledgments}

I would like to thank my supervisor Dr. Richard Mathias who provided invaluable insight and feedback during the preparation of this manuscript.

\section{References}

1. Public Health Agency of Canada and the Canadian Institue for Health Information. Obesity in Canada. Public Health Agency of Canada; 2011.

2. Mozaffarian D, Hao T, Rimm EB, Willett WC, Hu FB. Changes in diet and lifestyle and long-term weight gain in women and men. $\mathrm{N}$ Engl J Med. 2011;364(25):2392-404.

3. Institute of Medicine Food and Nutrition Board Standing Committee on the Scientific Evaluation of Dietary Reference Intakes. Dietary reference intakes for energy, carbohydrate, fiber, fat, fatty acids, cholesterol, protein and amino acids (Macronutrients) [Internet]. Washington (DC): The National Academies Press; 2005 [cited 2012 Apr 14]. Available from: http://www. ncbi.nlm.nih.gov/pubmed/12449285

4. Stanhope KL. Role of fructose-containing sugars in the epidemics of obesity and metabolic syndrome. Ann Rev Med. 2012 Feb 18;63(1):329-43.

5. Volek JS, Phinney SD. The Art and Science of Low Carbohydrate Living. Beyond Obese, LLC; 2011.

6. Gutierrez DA, Puglisi MJ, Hasty AH. Impact of increased adipose tissue mass on inflammation, insulin resistance, and dyslipidemia. Curr Diab Rep. 2009;9(1):26-32.

7. Choi SH, Ginsberg HN. Increased very low density lipoprotein (VLDL) secretion, hepatic steatosis, and insulin resistance. Trends Endocrinol. Metab. 2011 Sep;22(9):353-63.

8. Krauss RM, Blanche PJ, Rawlings RS, Fernstrom HS, Williams PT. Separate effects of reduced carbohydrate intake and weight loss on atherogenic dyslipidemia. Am. J. Clin. Nutr. 2006 May;83(5):1025-1031; quiz 1205.

9. Kristensen $\mathrm{M}$, Jensen $\mathrm{MG}$. Dietary fibres in the regulation of appetite and food intake. Importance of viscosity. Appetite. $2011 \mathrm{Feb} ; 56(1): 65-70$.

10. Siri-Tarino PW, Sun Q, Hu FB, Krauss RM. Meta-analysis of prospective cohort studies evaluating the association of saturated fat with cardiovascular disease. Am J Clin Nutr. 2010;91(3):535.

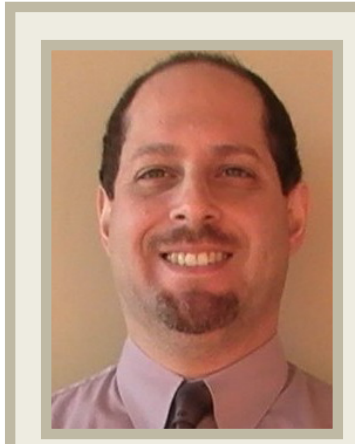

\section{Alexander Frame}

Alexander Frame holds a Medical Degree from the University of Sint Eustatius School of Medicine, a Bachelor of Science degree in Kinesiology, a Post-Baccalaureate Diploma in Gerontology and a Certificate in Applied Nutrition from Simon Fraser University. Currently, he is a MHSc candidate in Clinical Epidemiology at the University of British Columbia within the School of Population and Public Health. Dr. Frame's thesis research focuses on developing new clinical guidelines for the management of obesity and metabolic syndrome based on current evidence based medicine from the perspective of family and public health physicians. His research interests include health promotion and nutrition, geriatric medicine, and public health policy addressing disparities in health. 